RECYT

Año 22 / № 33 / 2020 / 27-32

\title{
Nuevo enfoque para el análisis energético-económico en la selección de las conductoras de impulsión
}

\section{New focus for the energy-economic analysis in the selection of the pressurized pipes}

\author{
José Luis Amador Vilariño', José Bienvenido Martínez Rodríguez², Oscar Brown Manrique ${ }^{3, *}$
}

1- Departamento de Mantenimiento. Instituto Nacional de Recursos Hidráulicos. Provincia de Camagüey.

2- Centro de Investigaciones Hidráulicas ( $\mathrm{ClH}$ ). Facultad de Ingeniería Civil. Universidad

Tecnológica de la Habana "José Antonio Echeverría". Cujae.

3- Centro de Estudios Hidrotécnicos (CEH). Facultad de Ciencias Técnicas. Universidad de Ciego de Ávila “Máximo Gómez Báez".

*E-mail: obrown@unica.cu

Recibido el 10 de julio de 2018, Aprobado el 11 de octubre de 2019.

\section{Resumen}

La investigación se realizó en tres obras hidráulicas: la estación de bombeo de la presa Máximo, la Potabilizadora de Camagüey y la estación de bombeo de la presa Pontezuela. Los resultados obtenidos demostraron que el método propuesto para el diseño de conductoras provocó una disminución de la potencia de las bombas y del costo de operación en $30,2 \%$ y 29,2 respectivamente y un tiempo de amortización promedio de 6,51 años. Los valores de velocidades más económicas estuvieron entre $0,52 \mathrm{~m} \mathrm{~s}^{-1}$ a 0,79 $\mathrm{m} \mathrm{s}^{-1}$ con un promedio de $0,655 \mathrm{~m} \mathrm{~s}^{-1}$. Los diseños hidráulicos de conductoras con reducción del diámetro para alcanzar la presión requerida en la descarga de la bomba; generan pérdidas superiores en casi dos veces la potencia necesaria en los motores de las electrobombas debido a que constituye un caso de estrangulación de la corriente; por lo que en caso de ser necesario esta variante se debe utilizar una válvula para que las pérdidas sean relativamente más bajas.

Palabras clave: Velocidad de diseño; Diámetro económico; Pérdidas de carga; Energía.

\section{Abstract}

The research was carried out in three hydraulic projects: the pumping station of Maximum dam, Camagüey Water treatment and the Pontezuela dam. The obtained results demonstrated that the proposed method for pipes design resulted on a power pumps decrease, cost operation reduction to $30,2 \%$ and $29,2 \%$ respectively and time amortization average within 6,51 year- time. The cheapest speed values were among $0,52 \mathrm{~m} \mathrm{~s}^{-1}$ to $0,79 \mathrm{~m} \mathrm{~s}^{-1}$ with an average of $0,66 \mathrm{~m} \mathrm{~s}^{-1}$. The hydraulic conductor designs with the necessary diameter reduction to reach the required pressure in pump discharge, generate higher losses in almost twice the times the needed power in the electric pump motors because it constitutes a case of strangling of the current. So, if this variant is necessary, a valve should be used in order to relatively reduce the losses.

Keywords: Speed design; Economic diameter; Charge losses; Energy.

\section{Introducción}

En el diseño ingenieril, los ingenieros hidráulicos requieren frecuentemente realizar cálculos relacionados con la determinación del diámetro de las conductoras, donde el diámetro óptimo representa un aspecto que influye significativamente desde el punto de vista económicos y energéticos. En este tipo de problemas, es recomendable la utilización de software para lograr mayor rapidez y exactitud [1]. Además del diámetro, la selección de los materiales apropiados, la correcta instalación y el mantenimiento son elementos esenciales para el funcionamiento correcto [2,3]. Todos estos esfuerzos permiten disminuir la intensidad energética a partir del análisis de los aspectos relacionados con el agua y energía [4].
La determinación del diámetro económico ha sido tratada por diferentes autores como [5,6,7,8,9]; pues generalmente se utiliza la ecuación de Bresse que no considera los costos de operación, por lo que los diámetros son relativamente elevados y las velocidades del flujo inferiores a 1,0 $\mathrm{m} \mathrm{s}^{-1}$; sin embargo, el diámetro económico debe basarse en los costos de la energía de bombeo y en los de amortización de la inversión [10]; de lo contrario, los sistemas hidráulicos dejan de ser eficientes cuando utilizan excesivos recursos humanos, materiales y económicos [11]. En esto influye también, el envejecimiento de las tuberías, las cuales pueden mejorar su funcionamiento con la aplicación de nuevos métodos de rediseño acorde con el desarrollo tecnológico actual [12]. 
En Cuba los criterios económicos siguen quedando en un segundo plano en comparación con los técnicos, a la hora de tomar decisiones relacionadas con las variantes de proyecto y con la recuperación de las inversiones que se realizan [13] y en el diseño de tuberías se establece como requisito que la velocidad de circulación en las tuberías de la ruta crítica no sobrepase los $1,5 \mathrm{~m} \mathrm{~s}^{-1}$ sin que este esté basado ningún análisis económico realizado en el país [14].

En el diseño de conductoras es necesario realizar la evaluación del riesgo y formular programas de mejoramiento que formen parte de las estrategias de gestión del riesgo [15]. Todo lo expuesto anteriormente permite considerar como objetivo del trabajo proponer un nuevo enfoque para el análisis energético-económico en la selección de las conductoras de impulsión utilizadas en los sistemas hidráulicos.

\section{Materiales y Métodos}

La investigación se basó en el diagnóstico hidráulico y energético realizado en las tres obras hidráulicas siguientes: (a) los proyectos de la estación de bombeo de la presa Máximo para el abastecimiento de agua a la ciudad de Camagüey; (b) la operación de la Potabilizadora de Camagüey, donde se abren y cierran diariamente válvulas de $1000 \mathrm{~mm}$ para que las bombas de $250 \mathrm{~kW}$ trabajen en los parámetros de funcionamiento establecidos y (c) el proyecto de la de la estación de bombeo de la presa Pontezuela a partir del uso de electro-bombas con presión de trabajo de $90 \mathrm{~m}$.

Los cálculos hidráulicos se realizaron mediante un programa desarrollado en esta investigación denominado "SISTELEC" basado en la metodología propuesta por [16].

En el análisis económico se tuvieron en cuenta los aspectos siguientes:

- Los precios de piezas, válvulas, bombas, grupos electrógenos, arrancadores, cables y transformadores se obtuvieron de los siguientes documentos: facturas de Cuba Hidráulica Provincial y Nacional; catálogos y listados de precios del Departamento de Presupuestos; precios de la Organización Básica Eléctrica y los ofrecidos por [17] y [18] para el combustible fósil y las fuentes renovables de energías.

- En el costo de la materia prima del PEAD (actualizado por Cuba Hidráulica Nacional) se consideró el costo de electricidad, que es de $698 \mathrm{~kW} . \mathrm{h} \mathrm{t}^{-1}$ con un $4,5 \%$ por el seguro de flete.

- Todos los costos de inversión adquiridos en el exterior, se incrementaron por el interés por créditos; usándose el $8 \%$ a pagar en 5 años.

- El costo de la energía eléctrica en kW.h en las líneas de 13,8 y $34,5 \mathrm{kV}$ se tomaron de la Unión Nacional Eléctrica con un valor de 0,15 USD.

- Los cálculos de costo-beneficio de las estaciones de bombeo se realizaron en una sola moneda (Dólares norteamericano, USD) para la energía, los precios de las tuberías, el equipamiento y las materias primas que el país tiene que adquirir en el mercado internacional.

Las variantes consideradas en el análisis general de las conductoras con un caudal de $3001 \mathrm{~s}^{-1}$, longitud de $30 \mathrm{~km}$, tiempo de vida útil de 20 años y tiempo de bombeo de $24 \mathrm{~h}$ al día durante todos los días del año fueron las siguientes:

-A: 1x500 mm: Una conductora de 500 mm de diámetro, proyectada por el método tradicional.

- B: 1x800 mm: Una conductora de 800 mm de diámetro, diseñada por el método propuesto.

- C: 1x900 mm: Una conductora de 900 mm de diámetro, diseñada por el método propuesto.

Las variantes para el análisis de las conductoras de la estación de bombeo de la presa Máximo para un caudal de $5501 \mathrm{~s}^{-1}$, tiempo de vida útil de 20 años y tiempo de bombeo de $24 \mathrm{~h}$ al día durante todos los días del año fueron las siguientes:

- D: 1x800 mm (P-I): Una conductora de $800 \mathrm{~mm}$ de diámetro proyectada por el método tradicional y construida; tiene una longitud de $17,8 \mathrm{~km}$ desde la presa Máximo hasta una inyección hecha en una tubería vieja de $1000 \mathrm{~mm}$ de hormigón pre-tensado.

- E: 2x800 mm (P-Pot): Dos conductoras de PEAD en paralelo de $800 \mathrm{~mm}$ de diámetro, diseñada por el método propuesto; tiene una longitud de 35,9 km desde la presa Máximo hasta la Potabilizadora pasando por la inyección.

- F: 2x900 mm (P-Pot): Dos conductoras de PEAD en paralelo de $900 \mathrm{~mm}$ de diámetro, diseñada por el método propuesto; tiene una longitud de 35,9 km desde la presa Máximo hasta la Potabilizadora pasando por la inyección.

- G: 2x1000 mm (P-Pot): Dos conductoras de PEAD en paralelo de $1000 \mathrm{~mm}$ de diámetro, diseñada por el método propuesto; tiene una longitud de 35,9 km desde la presa Máximo hasta la Potabilizadora pasando por la inyección en la conductora de hormigón.

- H: 2x800 mm (P-I) + 2x900 (P-Pot): Dos conductoras en paralelo de $800 \mathrm{~mm}$ de diámetro, diseñada para ser construida y resolver el problema existente; tiene una longitud de 17,8 km desde la Presa Máximo hasta la inyección en la conductora de hormigón, acoplada a dos conductoras en paralelo de $900 \mathrm{~mm}$ de diámetro interior y longitud de 35,9 km desde la presa Máximo hasta la Potabilizadora pasando por la inyección. en la conductora de hormigón.

- I: 2x800 mm (P-I) + 1x1000H (I-Pot): Dos conductoras en paralelo de $800 \mathrm{~mm}$ de diámetro, diseñada para ser construida y resolver el problema existente; tiene una longitud de $17,8 \mathrm{~km}$ desde la presa Máximo hasta la inyección en la conductora de hormigón, acoplada, además a la conductora de $1000 \mathrm{~mm}$ de diámetro interior de hormigón existente con una distancia de $18,1 \mathrm{~km}$ hasta la Potabilizadora. 
Las variantes evaluadas en el análisis de las conductoras de la estación de bombeo de la presa Pontezuela para un caudal de $7451 \mathrm{~s}^{-1}$, longitud de $22 \mathrm{~km}$, tiempo de vida útil de 20 años y 92 días de bombeo al año fueron las siguientes:

- J: 1x800 mm: Una conductora de $800 \mathrm{~mm}$ de diámetro, diseñada por el método propuesto para la estación de bombeo de la presa Pontezuela.

- K: 1x1000 mm: Una conductora de $1000 \mathrm{~mm}$ de diámetro, diseñada para ser construida y resolver el problema existente en la conductora de la estación de bombeo de la presa Pontezuela.

La selección de la variante más económica a partir del análisis por anualidades se realizó por la ecuación propuesta por [19], la cual se escribe como:

$$
\begin{aligned}
& C_{T}=I+C_{A} \cdot n \\
& G_{O}=C_{B}=C_{A} \cdot n
\end{aligned}
$$

Donde:

$C_{T}$ es el costo total; $I$ la inversión; $C_{A}$ el costo anual; $n$ el periodo de viva útil; $G_{O}$ el gasto de operación; $C_{B}$ el costo de bombeo.

El cálculo de las anualidades se realizó con el software CALCO desarrollado también en esta investigación en el cual se incluyen el costo total equivalente y el costo anualizado total que se calculan según [19] mediante las ecuaciones siguientes:

$$
C A T=\frac{r(1+r)^{20 n}-1}{(1+r) n-1} I+C_{A}
$$

Donde CAT es el costo anualizado total; $r$ la tasa de interés bancaria; $n$ el período de vida útil; $I$ la inversión inicial; $C_{A}$ el costo anual.

\section{Resultados y Discusión}

En la tabla 1 se presentan los resultados de la selección de variantes económicas, en función de la potencia que entra por los cables del motor $\left(P_{e n t}\right)$; la diferencia del costo de la energía del bombeo con respecto a la energía de bombeo del método tradicional para el periodo de un año $\left(\Delta \mathrm{CE}_{a \tilde{n} o}\right)$; la diferencia del costo de la energía total con respecto a la calculada por el método tradicional para el periodo de 20 años $\left(\Delta \mathrm{CE}_{20 \text { años }}\right)$; la velocidad del flujo $(v)$; la carga total $\left(H_{t}\right)$ y el porcentaje de carga total con respecto a la carga estática de $30 \mathrm{~m}\left(\mathrm{PH}_{\mathrm{t}}\right)$.

En la variante A, donde se aplicó el método tradicional se obtuvo un diámetro real de $455 \mathrm{~mm}$ y velocidad de $1,86 \mathrm{~m} \mathrm{~s}^{-1}$, que está comprendida dentro de las "normales" especificada en la norma cubana NC-53-121/84 donde esta variable puede variar desde $1,2 \mathrm{~m} \mathrm{~s}^{-1}$ hasta $1,8 \mathrm{~m} \mathrm{~s}^{-1}$.

Los mejores resultados se lograron en la variante $\mathrm{C}$ donde se aplicó el método propuesto con un diámetro de
$900 \mathrm{~mm}$ por tener las mayores diferencias positivas del costo de la energía de bombeo. En este caso la diferencia de potencias de los motores eléctricos fue de $693 \mathrm{~kW}$.

En la tabla 2 se presentan los resultados de la selección de variantes económicas para el diseño de la conductora de la estación de bombeo Máximo en función de las variables explicadas en la tabla1, con la incorporación del tiempo de amortización con respecto a la conductora construida $\left(T_{A}\right) \mathrm{y}$ la ganancia con respecto a la conductora construida en un período de 20 años $\left(G_{20 a n ̃ o s}\right)$. Se puede observar que, en la variante $\mathrm{D}$, la carga total de la tubería actual instalada con diámetro de $800 \mathrm{~mm}$ es de 102,2 m; sin embargo, con la adición por parte del proyectista de $44 \mathrm{~m}$ adicionales para lograr la supuesta presión necesaria en la tubería de la estación de bombeo cubano-búlgara se llegó al valor de 146 m. Esta solución no es favorable; porque requiere del estrangulamiento de las válvulas en la descarga de las bombas para reducir la presión de $146 \mathrm{~m}$ hasta 102,2 m; lo que implica un gasto de energía innecesaria por no haberse hecho un análisis correcto.

En la propia tabla 2 se comprueba que existen grandes diferencias de potencias entre la variante D y el resto de las variantes analizadas. En esta variante la potencia es de $1215 \mathrm{~kW}$, lo que representa un consumo de energía superior en el rango de 1,7 a 2,2 veces. La variante E resultó ser la más económica dentro de las conductoras diseñadas con la metodología propuesta. Esto responde al hecho de que se obtuvo la mayor ganancia y el menor tiempo de amortización respecto a la conductora construida.

La variante más económica de forma absoluta se encontró en la variante I consistente en una propuesta para la reconstrucción adecuada del proyecto ya ejecutado. El uso de dos tuberías de $800 \mathrm{~mm}$ en lugar de una permite reducir significativamente la fricción. Con esta variante se alcanza una diferencia de potencia y de carga total de $549,00 \mathrm{~kW} \mathrm{y}$ $58,20 \mathrm{~m}$ respectivamente.

Tabla 1: Análisis general de conductoras con diferentes diámetros.

\begin{tabular}{|c|c|c|c|c|c|c|c|}
\hline Variantes & $\begin{array}{l}P_{\text {ent }} \\
(\mathrm{kW})\end{array}$ & $\begin{array}{l}\Delta \mathrm{CE}_{\text {año }} \\
\text { (USD) }\end{array}$ & $\begin{array}{c}\mathrm{T}_{\mathrm{A}} \\
\text { (años) }\end{array}$ & $\begin{array}{l}\mathbf{G}_{20 \tilde{o s}} \\
\text { (USD) }\end{array}$ & $\begin{array}{c}\mathbf{v} \\
\left(\mathrm{m} \mathrm{s}^{-1}\right)\end{array}$ & $\begin{array}{l}\mathrm{H}_{\mathrm{t}} \\
(\mathrm{m})\end{array}$ & $\begin{array}{l}\mathrm{PH}_{\mathrm{t}} \\
(\%)\end{array}$ \\
\hline \multicolumn{8}{|c|}{ Proyectada por el método tradicional y construida } \\
\hline $\begin{array}{l}\text { D: } 1 \text { X800 } \\
\mathrm{mm}(\mathrm{P}-\mathrm{I})\end{array}$ & 1215,00 & - & - & - & 1,20 & 146,00 & 208,00 \\
\hline \multicolumn{8}{|c|}{ Diseñada por el método propuesto } \\
\hline $\begin{array}{l}\text { E: 2x800mm } \\
\text { (P-Pot) }\end{array}$ & 662,30 & 590362,00 & 4,49 & 7381675,00 & 0,68 & 86,15 & 123,00 \\
\hline $\begin{array}{l}\text { F: 2x900mm } \\
\text { (P-Pot) }\end{array}$ & 606,90 & 650429,00 & 13,90 & 4454024,00 & 0,60 & 79,18 & 113,00 \\
\hline $\begin{array}{l}\text { G: } \\
1 \times 1000 \mathrm{~mm} \\
\text { (P-Pot) }\end{array}$ & 698,00 & 541938,00 & 6,70 & 7204393,00 & 0,85 & 90,49 & 129,00 \\
\hline Diferencia & 552,70 & & & & & & \\
\hline
\end{tabular}

\begin{tabular}{|c|c|c|c|c|c|c|}
\hline Variantes & $\begin{array}{c}\mathbf{P}_{\text {ent }} \\
(\mathbf{k W})\end{array}$ & $\begin{array}{c}\Delta \mathrm{CE}_{\text {año }} \\
(\mathbf{U S D})\end{array}$ & $\begin{array}{c}\Delta \mathrm{CE}_{20 \text { añso }} \\
(\mathbf{U S D})\end{array}$ & $\begin{array}{c}\mathbf{V} \\
\left(\mathbf{m ~ s}^{-1}\right)\end{array}$ & $\begin{array}{c}\mathbf{H}_{\mathrm{t}} \\
(\mathbf{m})\end{array}$ & $\begin{array}{c}\mathbf{P H}_{\mathbf{t}} \\
(\%)\end{array}$ \\
\hline \multicolumn{7}{|c|}{ Diseñada por el método tradicional } \\
\hline A: $1 \times 500 \mathrm{~mm}$ & 847,30 & - & - & 1,86 & 212,02 & 706,00 \\
\hline \multicolumn{7}{|c|}{ Diseñada por el método propuesto } \\
\hline B: $1 \times 800 \mathrm{~mm}$ & 180,18 & 867342,00 & 18871270,00 & 0,68 & 45,09 & 150,00 \\
\hline C: $1 \times 900 \mathrm{~mm}$ & 153,34 & 902847,00 & 18984730,00 & 0,52 & 37,95 & 126,50 \\
\hline Diferencia & 693,96 & & & & & \\
\hline
\end{tabular}

Tabla 2: Diseño de la conductora de la estación de bombeo Máximo. 


\begin{tabular}{|c|c|c|c|c|c|c|c|}
\hline Variantes & $\begin{array}{c}P_{\text {ent }} \\
(\mathrm{kW})\end{array}$ & $\begin{array}{l}\Delta \mathrm{CE}_{\text {año }} \\
\text { (USD) }\end{array}$ & $\begin{array}{c}T_{A} \\
\text { (años) }\end{array}$ & $\begin{array}{l}\mathbf{G}_{20 \text { กัos }} \\
\text { (USD) }\end{array}$ & $\begin{array}{c}\mathbf{v} \\
\left(\mathrm{m} \mathrm{s}^{-1}\right)\end{array}$ & $\begin{array}{r}\mathbf{H}_{\mathrm{t}} \\
(\mathrm{m})\end{array}$ & $\begin{array}{l}\mathrm{PH}_{\mathrm{t}} \\
(\%)\end{array}$ \\
\hline \multicolumn{8}{|c|}{ Diseñada para ser construida y resolver el problema existente } \\
\hline \multicolumn{8}{|c|}{ H: 2X800mm (P-I) + 2X900mm (I-Pot) } \\
\hline & 636,00 & 625710,00 & 3.87 & 10091762,00 & $\begin{array}{l}0,64- \\
0,51\end{array}$ & 82,40 & 117,00 \\
\hline \multicolumn{8}{|c|}{ I: $2 \times 800$ mm (P-I) + 1X1000H (I-Pot } \\
\hline & 666,00 & 570003,00 & 1,15 & 10755408,00 & $\begin{array}{c}0,64- \\
0,79 \\
\end{array}$ & 87,80 & \\
\hline Diferencia & 549,00 & & & & & 58,20 & \\
\hline
\end{tabular}

En la figura 1 se muestra la curva de potencia en $\mathrm{kW}$ contra caudal en $1 \mathrm{~s}^{-1}$ de las bombas centrifugas instaladas en la estación de bombeo de la presa Máximo, en la que se observa que la potencia que se entrega para $1151 \mathrm{~s}^{-1}$ y 146 $m$ de carga es de $215 \mathrm{~kW}$ en cada una de las cinco bombas de $1101 \mathrm{~s}^{-1}$; sin embargo, en la variante I $(2 \times 800 \mathrm{~mm}+$ $1 \mathrm{x} 1000 \mathrm{H})$ la potencia de la bomba no es superior a 130 $\mathrm{kW}$ en cada una de ellas.

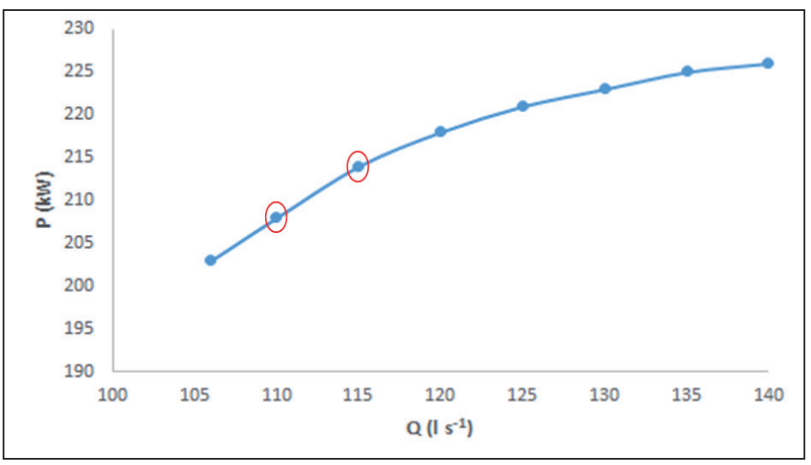

Figura 1: Curva de potencia - caudal.

Si se hace una comparación entre las cinco bombas que debieron haberse seleccionado con carga de $102 \mathrm{~m}$ y caudal de $1101 \mathrm{~s}^{-1}$ respecto a las que se instalaron con carga de $146 \mathrm{~m}$ y caudal de $115 \mathrm{l} \mathrm{s}^{-1}$, se observa que es necesario el estrangulamiento para garantizar la protección de los motores. Esta operación equivale para las cinco bombas estranguladas, utilizar un motor con una potencia superior a $315 \mathrm{~kW}$, acoplado a un reductor que trabaja las 24 horas del día. Como consecuencia de este diseño insatisfactorio, la Empresa de Acueductos de la provincia de Camagüey determinó en marzo del 2009, utilizar la estación de bombeo de Máximo sólo para emergencias.

En la tabla 3 se presentan los resultados de la selección de variantes económicas para el diseño de la conductora de la estación de bombeo de la presa Pontezuela en función de las mismas variables consideradas en la tabla 2; pero con la inclusión del tiempo de bombeo anual $\left(T B_{a}\right)$ expresado en días por año. Este caso representa el diseño de estaciones de bombeo con sus conductoras donde se hacen adaptaciones de tuberías a las bombas, cuando en realidad debe ser siempre al revés.
Tabla 3: Parámetros del diseño de la conductora de la estación de bombeo de la presa Pontezuela.

\begin{tabular}{|c|c|c|c|c|c|c|c|}
\hline Variantes & $\begin{array}{c}\boldsymbol{P}_{\text {ent }} \\
\text { (kW) }\end{array}$ & $\begin{array}{l}\Delta C E_{\text {año }} \\
\text { (USD) }\end{array}$ & $\underset{\text { (años) }}{T_{A}}$ & $\begin{array}{l}\boldsymbol{G}_{\text {20ños }} \\
\text { (USD) }\end{array}$ & $\begin{array}{c}\boldsymbol{v} \\
\left(\mathrm{m} \mathrm{s}^{-1}\right)\end{array}$ & $\begin{array}{c}\boldsymbol{H}_{t} \\
(\mathrm{~m})\end{array}$ & $\begin{array}{c}T B_{a} \\
\text { (días año-1) }\end{array}$ \\
\hline \multicolumn{8}{|c|}{ Proyectada por el método tradicional y construida } \\
\hline $\begin{array}{c}\mathrm{J}: 1 \times 800 \\
\mathrm{~mm}\end{array}$ & 930,00 & - & - & - & 1,80 & 90,00 & 92,00 \\
\hline \multicolumn{8}{|c|}{ Diseñada para ser construida y resolver el problema existente } \\
\hline 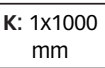 & 511,00 & 139120,00 & 1,28 & 2603000,00 & 1,07 & 48,00 & 92,00 \\
\hline Diferencia & 419,00 & & & & & 42,00 & \\
\hline
\end{tabular}

El análisis de este diseño indica que, para el caudal transportado y la cantidad de días de funcionamiento, el diámetro económico de la tubería debió ser de $1000 \mathrm{~mm}$ y no de $800 \mathrm{~mm}$, como realmente fue proyectada (variante J), lo que incrementa innecesariamente el gasto de operación. La variante $K$ es la más económica al lograr una diferencia favorable de $419 \mathrm{~kW}$ de potencia y $42 \mathrm{~m}$ de carga total.

En la figura 2 se muestran los resultados del cálculo económico para diferentes variantes de diseño a partir del software CALCO, lo que le permite al proyectista seleccionar el mejor resultado teniendo en cuenta los costos de inversión, operación y totales que ofrece este programa con mucha rapidez y confiabilidad.

En la figura 3 se presentan los resultados del análisis por anualidades a partir del cual se comprobó que el caso de análisis general de las conductoras, los resultados más económicos se encontraron en la variante $\mathrm{C}$ con caudal de $3001 \mathrm{~s}^{-1} \mathrm{y}$ diámetro de $900 \mathrm{~mm}$ de PEAD.

En este cálculo se empleó una tasa baja de interés bancario de 3\%, la cual es utilizadas por [19]; debido a que no existe un mecanismo explícito de recuperación de la inversión a través del cobro del agua que entrega la obra y también a que las inversiones que se realizan actualmente por el Instituto Nacional de Recursos Hidráulicos, se hacen adquiriendo el dinero en el exterior o en una entidad donadora.

\section{Conclusiones}

Se demostró que el método propuesto para el diseño de conductoras logró disminuir la potencia instalada en $\mathrm{kW}$ en un $30,2 \%$ y los costos de operación en un $29,2 \%$ para todas las variantes más económicas, con respecto al diseño con el método tradicional; mientras que se logró que el tiempo de amortización promedio fuera de 6,51 años.

Se obtuvieron valores de velocidades más económicas entre $0,52 \mathrm{~m} \mathrm{~s}^{-1}$ a $0,79 \mathrm{~m} \mathrm{~s}^{-1}$ con un promedio de $0,655 \mathrm{~m} \mathrm{~s}^{-1}$.

Los diseños hidráulicos de conductoras al revés constituye un caso de estrangulación; por lo que de ser necesario su realización, debe hacerse con una válvula para que el valor de las pérdidas sea relativamente bajo. 


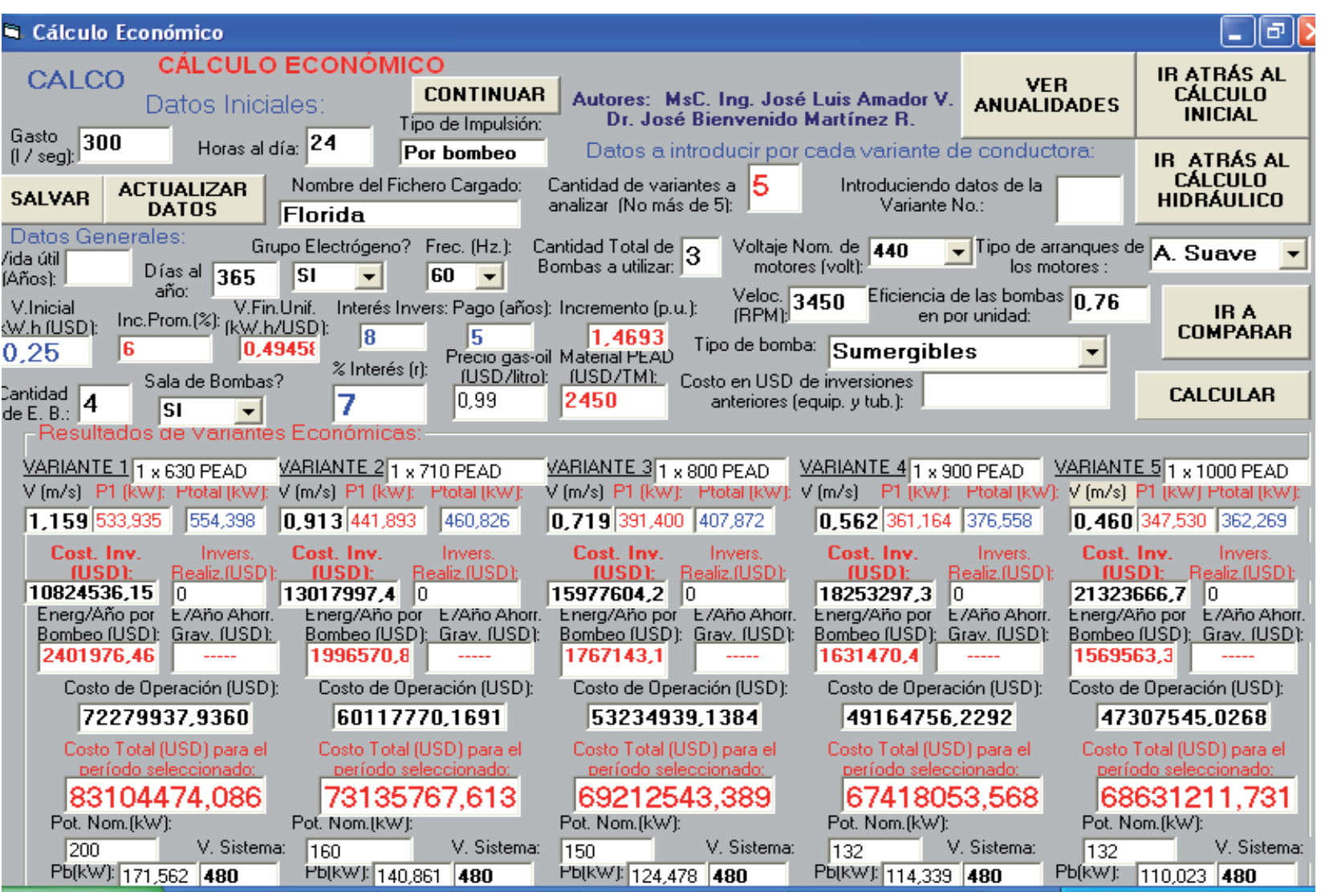

Figura 2: Cálculo económico con el programa CALCO.

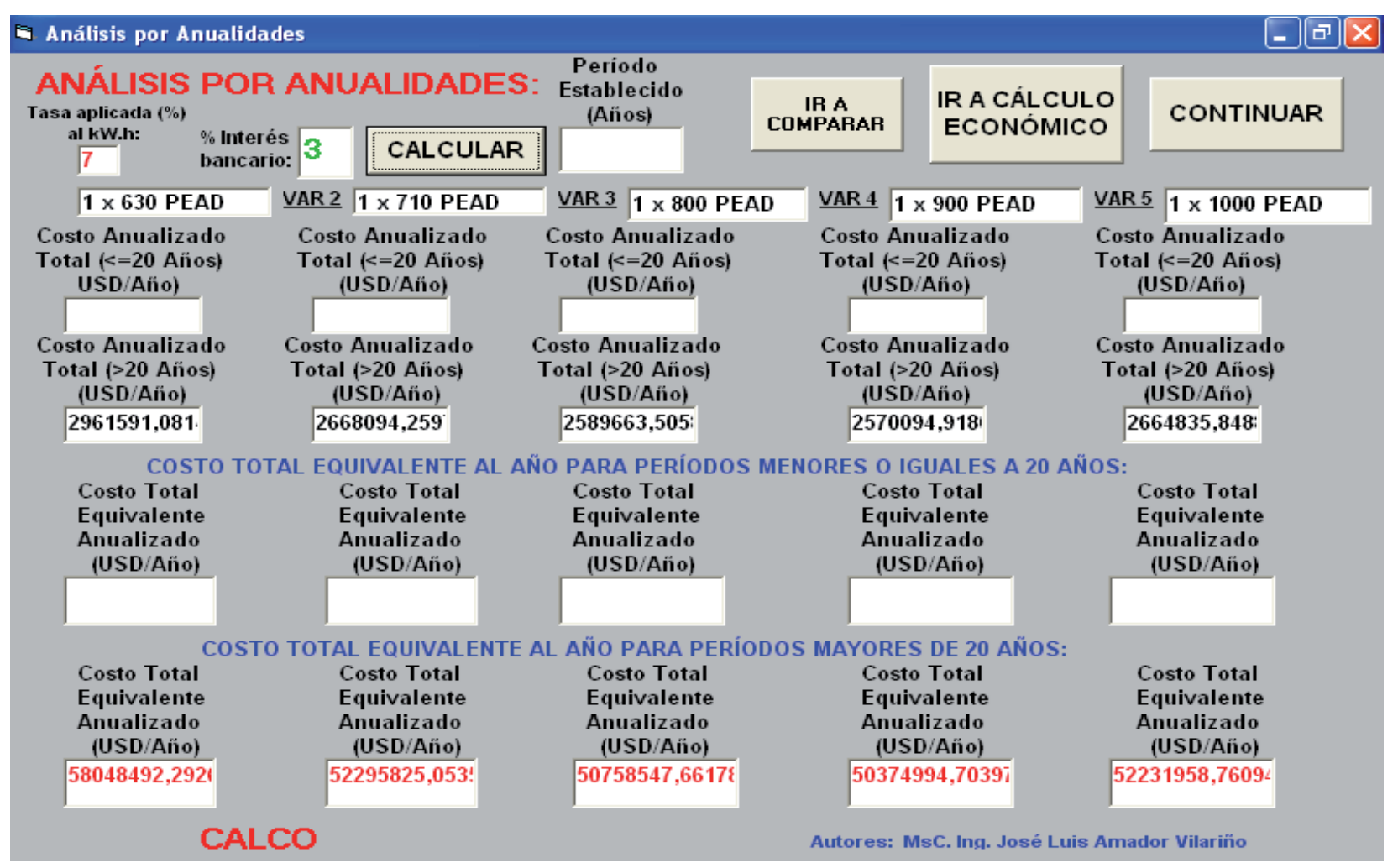

Figura 3: Análisis por anualidades con el programa CALCO. 


\section{Referencias}

1. García, J. y Morales, A. Determinación del diámetro en sistemas de tuberías utilizando el Mathcad. Revista Ingeniería, Vol.7, no 1, pp. 53-58. ISSN: 1665-529X. México, 2003.

2. Nakamura, y. Estudio económico de distintos tipos de conducciones de aguas. Boletín Técnico INIA No. 44. ISBN: 0717-4829. Chile, 2000.

3. Stuyt, L.C., Dierickx, w. y Martínez, J. Materiales para sistemas de drenaje subterráneo. Estudio FAO Riego y Drenaje 60. ISSN: 978-92-5-305427-5. Roma, Italia, 2009.

4. IDAE. Ahorro y Eficiencia Energética en Agricultura de Regadio. Serie Ahorro y Eficiencia Energética en la Agricultura. ISBN: 84-86850-94-0, Madrid, España, 2005.

5. Blanco, E., Velarde, s. y Fernández, J. Sistemas de bombeo. Universidad de Oviedo, E. T. S. Ingenieros Industriales. Departamento de Energía, pp.217. ISBN 84-6049677-5, España, 1994.

6. Roldán, J., Pulido, I., Camacho, E., Alcaide, M. y Losada, A. Problemas de hidráulica para riego. Universidad de Córdoba. ISBN: 84-7801-526-4, pp.284. España, 1999.

7. Pedrevello, C.L. Diâmetro mais econômico de uma canalização de recalque. Revista Brasileira de Recursos Hídricos, Vol.5, no 2, pp. 39-42. ISSN: 2318-0331. Brasil, 2000.

8. Carnicer, E. y Mainar, C. Bombas centrifugas, Segunda edición, Thomson Editores Spain, Paraninfo, S.A, ISBN: 84-283-2243-0, España, 2004.

9. González, H. y Suarez, A.E. Hidrología e hidráulica para ingenieros civiles. Editorial Félix Varea, ISSN: 978959-07-1680-5, pp.436, La Habana, Cuba, 2012.

10. Comisión Nacional del Agua. Manual de agua potable, alcantarillado y saneamiento. Cárcamos de bombeo para alcantarillado, funcional e hidráulico. Editor: Secretaría de Medio Ambiente y Recursos Naturales, ISBN: 978-968-817-880-5, México, 2007.
11. Comisión Nacional del Agua. Manual de Incremento de la ciencia Física, Hidráulica y Energética en Sistemas de Agua Potable. ISBN: 978-607-7908-68-5, pp.175, Tlalpan, México, 2012.

12. Iñiguez, M., ojeda, w. y Díaz, c. Mejoras de eficiencia hidráulica en vertedores con canal de descarga libre en presas: propuesta metodológica. Tecnología y Ciencias del Agua, Vol.6, no 1, pp. 69-79. ISSN: 0187-8336. México, 2015.

13. Pérez, L.R., Sánchez, R.M. y Frizzone, J.A. Optimal moment to change pressure regulator and sprayer kit on center pivot irrigation machines: application to a study case". IRRIGA. Brazilian Journal of Irrigation and Drainage. Vol.16, no 4, pp. 450-458. ISSN: 1808-8546, Brasil, $2011 \mathrm{a}$.

14. Pérez, R., Soto, S.D. y Conde, P. El diseño de sistemas de riego localizado a partir de un nuevo criterio de velocidad. Ingeniería Agrícola. Vol.6, no 3, pp. 48-53. ISSN: 2306-1545. Cuba, 2016b.

15. Amézquita, C.P., Pérez, A. y Torres, P. Evaluación del riesgo en sistemas de distribución de agua potable en el marco de un plan de seguridad del agua. Escuela de Ingeniería de Antioquia (EIA). Revista EIA. Vol.11, no 21, pp. 157-169. ISSN: 1794-1237. Colombia, 2014.

16. Pérez, D. Estaciones de bombeo. Editorial Félix Varela, ISBN: 978-959-07-1379-8, pp.295, La Habana, Cuba, 2011.

17. Bluejay, м. How much does electricity cost. What is a kilowatt-hour (kWh)". [en línea] 2011, Disponible en: http:/michaelbluejay.com/electricity/ [Consulta: 28 de diciembre de 2017].

18. Rodríguez, J.L. La disminución de la alta dependencia del combustible importado para la generación”. Cuba contemporánea. [en línea] 2012, Disponible en: http:// cubasi.cu/cubasi, [Consulta: 04 de enero de 2017].

19. Martínez, J.B. Economía de los Recursos Hidráulicos. Editorial Félix Varela, pp.245, La Habana, Cuba, 2000. 